PALEO

Revue d'archéologie préhistorique

30-1 | 2019

Varia

Jean Abélanet

(1925-2019)

\title{
Dominique Sacchi
}

\section{CpenEdition}

Journals

Édition électronique

URL : http://journals.openedition.org/paleo/4283

DOI : $10.4000 /$ paleo.4283

ISSN : 2101-0420

Éditeur

SAMRA

\section{Édition imprimée}

Date de publication : 30 décembre 2019

Pagination : 9-13

ISSN : $1145-3370$

Référence électronique

Dominique Sacchi, « Jean Abélanet », PALEO [En ligne], 30-1 | 2019, mis en ligne le 29 mai 2020, consulté le 07 juillet 2020. URL : http://journals.openedition.org/paleo/4283 ; DOI : https://doi.org/ $10.4000 /$ paleo.4283

\section{(ब) $\odot \Theta$}

PALEO est mis à disposition selon les termes de la licence Creative Commons Attribution - Pas d'Utilisation Commerciale - Pas de Modification 4.0 International. 


\section{JEAN ABÉLANET $(1925-2019)$}

Durant sa longue vie Jean Abélanet demeura attaché à sa terre natale et ne s'en éloigna guère. Né le 20 août 1925 à Rivesaltes il y décéda le 21 mars 2019. Après ses études secondaires au Petit Séminaire de Perpignan il intégra le Grand Séminaire et fut ordonné prêtre en 1948. Nommé professeur de lettres dans le premier de ces établissements, il exerça ensuite son sacerdoce comme vicaire de la paroisse de Céret, puis en tant que curé de Vernet-les-Bains. C'est dans cette petite cité thermale que je fis sa connaissance en 1967, sous la conduite de Jean et Christiane Guilaine. Ayant obtenu sa « reconduction à l'état laïque » sous le pontificat de Paul VI, à l'égard duquel il ne cessera d'exprimer sa profonde gratitude, il se maria en 1971. Le couple s'établit à Rivesaltes et donnera naissance à deux filles.

Selon ses propres termes, c'est en 1943, « au fond de la grotte de Montou [Corbère-les-Cabanes], que naquit, à l'initiative et à l'exemple de Pierre Ponsich, [sa] vocation de préhistorien » (Abélanet 1987c). Dès lors sa déférente estime pour l'érudit perpignanais, aux multiples centres d'intérêts, est définitivement acquise. Désireux d'approfondir ses connaissances, Jean Abélanet suivra l'enseignement de Maurice Louis, chargé de cours à la Faculté des Lettres de Montpellier et Directeur de la XI Circonscription des Antiquités Préhistoriques et se verra décerner, en 1949, un Certificat d'études supérieures en Préhistoire. Dans la foulée il entamera des recherches, autorisées aux termes de la récente loi sur les fouilles archéologiques, dans la grotte du Pas Estret à Opoul. Après la mise au jour de vestiges d'une occupation épipaléolithique (Sacchi 1986), il interrompit ses travaux en 1951, à la suite de l'immixtion destructrice d'un collectionneur. L'année suivante il découvrit des sépultures néolithiques dans La Cova de l'Esperit à Salses (Abélanet, Charles 1964a). Le remplissage de cette cavité contenait également des matériaux relatifs à une occupation mésolithique identifiée ultérieurement (Martzluff, Abélanet 1987 et 1989b). En 1954 il initiera des recherches dans la grotte d'Embulla à Corneilla-de-Conflent. Le niveau archéologique, initialement attribué au Magdalénien, se révélera appartenir au Solutréen supérieur après reconnaissance, par Philippe Smith, des pièces extraites clandestinement et illicitement emportées au Québec par l'individu responsable des dégâts commis au Pas Estret (Sacchi, Abélanet 1980, Sacchi 1986).

Cette nouvelle mésaventure l'affectera durablement et l'incitera à orienter son activité vers la prospection en plein air ce qui l'amènera à recueillir des outillages paléolithiques sur d'anciennes terrasses alluviales (Lumley et al. 1976) et à repérer de nombreux sites.

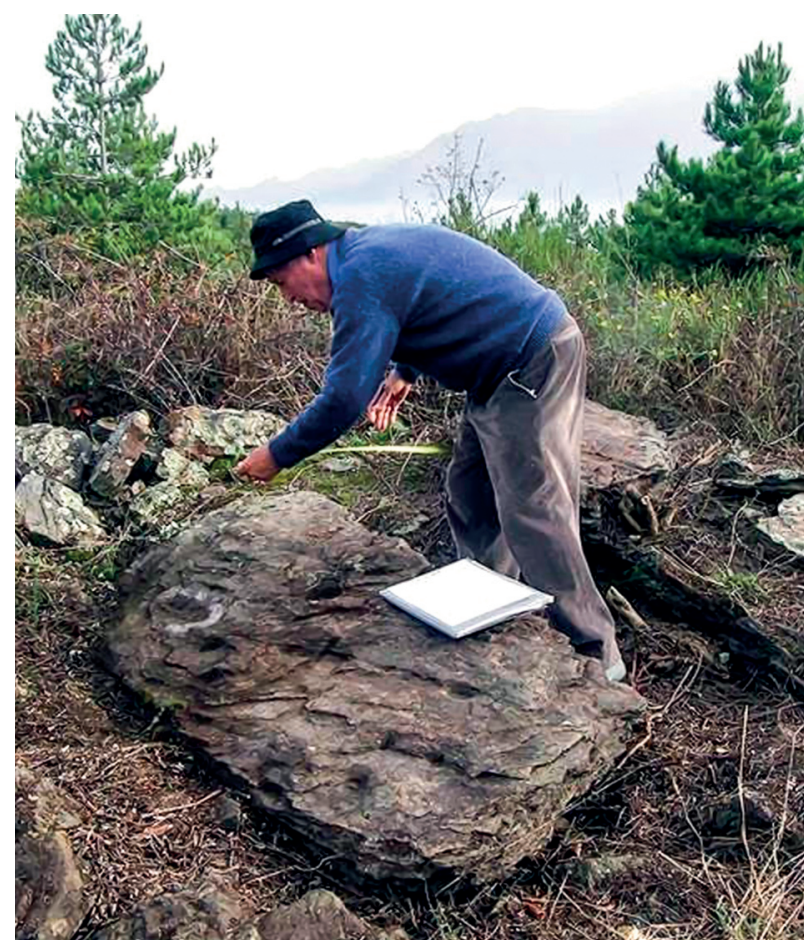

Jean Abélanet prenant des mesures sur la table du dolmen du Roc de l'homme mort, à Ria, Pyrénées-Orientales (photo M. Martzluff).

Notamment les stations magdalénienne de la Teulera à Tautavel, et solutréenne des Espasols 91 à Vingrau, dont il me confia le matériel pour étude (Sacchi 1986 et 1991). Mais c'est aux mégalithes et aux roches gravées qu'il consacrera l'essentiel de son temps libre. À cet égard sa situation oscillera entre celle de l'« amateur »- on dit aujourd'hui «chercheur bénévole », par euphémisme - et celle du professionnel dont il ne connaîtra véritablement le statut qu'en 1978. Cette année-là il accède au poste de conservateur du Musée de préhistoire de Tautavel, nouvellement créé (Abélanet 1982). Il occupera cette fonction jusqu'à sa retraite, en 1990, et en conservera un souvenir contrasté mêlé d'amertume. Auparavant, après une tentative de candidature au CNRS, il reçut, de 1968 à 1978, une allocation de recherche de cet organisme public. De 1975 à 1980 il enseigna la préhistoire au Centre universitaire de Perpignan devenu Université autonome en 1979. Ayant su captiver l'intérêt des étudiants, par son aptitude pédagogique, et du même coup éveiller celui du Conseil d'Université, la décision fut prise, en 1982, de pourvoir à la nomination d'un Maître de Conférence chargé de poursuivre l'enseignement d'une discipline, toujours professée à ce jour (Descamps 2005).

Bien qu'ayant fait la démonstration de ses qualités d'archéologue généraliste, il s'illustra tout particulièrement dans l'étude des dolmens en enrichissant considérablement leur inventaire, à l'échelle du département des Pyrénées-Orientales. La description de chacun de ces monuments et l'approche des rites funéraires donnèrent lieu à la publication d'un ouvrage que ses confrères le pressaient de produire (Abélanet 2011). Mais c'est sans doute dans l'étude des pétroglyphes protohistoriques, antiques et médiévaux, sujet de son mémoire de thèse (Abélanet 1977), qu'il trouva la manière 
de satisfaire au mieux son inclination pour les manifestations esthétiques et symboliques. Il acquit dans ce domaine une compétence unanimement reconnue qui le conduisit un temps à quitter son territoire familier pour diriger, à partir de 1967, quelques missions au Mont Bego. Cette expérience, qu' il décida d'interrompre, lui fournira cependant l'occasion d'établir la chronologie interne de ce haut lieu de l'art rupestre et, sur un plan plus général, d'élargir son champ d'observation et d'enrichir sa réflexion (Abélanet 1976a, b, c). Elle le confirmera en outre dans sa vocation de chercheur indépendant et confortera son inappétence pour l'exercice du pouvoir, fut-il limité au rôle de chef d'équipe. Pour répondre à l'amicale sollicitation de Jean Guilaine, il rédigera un ouvrage synthétique intitulé Signes sans paroles. Cent siècles d'art rupestre en Europe occidentale. Dans cette approche descriptive, chronologique et interprétative, clairement conçue et élégamment rédigée, Jean Abélanet donne toute la mesure de son talent, de son savoir et de son attrait constant pour le sacré.

C'est en explorant le Pla de Vall en So, en 1983, qu'il repéra la tête et l'arrière-train de ce qu'il pensait être un boviné, finement gravé sur le rocher de Fornols à Campôme (Abélanet 1985). Surpris par la nature du thème et le style de cette figure il m'invita à poser sur elle un diagnostic. Contre toute attente, il venait de découvrir deux segments d'une figure d'isard, et non de bœuf, indubitablement magdalénienne et, par la même occasion, l'unique décor paléolithique à l'air libre connu sur le sol de France. Il me laissa généreusement le soin d'en assurer le déchiffrage et l'étude sans accepter de s'y associer comme je l'en priais (Sacchi 1984, Sacchi et al. 1988). Il eut précédemment la même attitude lors de la révision du décor peint minimaliste de la Cova Bastéra à Corneilla-de-Conflent (Sacchi, Abélanet, Vilette 1984).

Dans un ultime ouvrage, il tira prétexte de sa rencontre avec le thème de la chasse au cerf, observé à diverses reprises sous la forme de décors rupestres, pour traiter, d'une manière que d'aucuns jugeront quelque peu syncrétiste, du symbolisme de cet animal souvent qualifié de psychopompe. Il s'agissait pour lui de revenir au phénomène religieux à travers les mythes, de convoquer les données collectées au fil des ans.

Il éprouvera de la satisfaction à voir Pierre Campmajo marcher sur ses traces dans l'entreprise de recensement systématique et de relevés descriptifs de l'art rupestre de Cerdagne (Campmajo 2012). Sans doute verra-t-il comme un accomplissement à sa démarche pionnière le lancement, en 2010, du programme d'étude, de gestion et de conservation de ce patrimoine vulnérable, piloté par le Service régional de l'Archéologie. Mais il manquera de peu l'ouverture de l'exposition Mémoires de pierres. Les gravures rupestres de Cerdagne et d'ailleurs au ChâteauMusée de Bélesta (Lallemand 2019), où il était attendu.

Par son ouverture aux autres et la qualité de ses observations, Jean Abélanet favorisa l'implantation de la première génération de chercheurs professionnels en Roussillon. Comme j'ai déjà eu l'occasion de le dire ailleurs en sa présence, il favorisa l'avènement des recherches programmées qui sonnaient le glas du temps révolu des travaux désordonnés commis par des collectionneurs. Soucieux d'assurer la conservation du produit de ses recherches il collabora à la création d'un dépôt archéologique départemental dont il fut un temps le responsable.

Tous ceux qui l'approchaient demeuraient sous le charme de son ineffable sourire. Mais derrière l'affabilité naturelle, faite de simplicité et d'élans chaleureux envers les êtres, de son admiration pour les œuvres du passé et leurs auteurs anonymes, il ne manquait pas de manifester avec force ses convictions. Sa fierté de découvreur, clairement revendiquée, tempérait parfois sa réelle modestie. L'hommage enthousiaste qui lui fut rendu en 2001 à l'Université de Perpignan, à l'initiative de Michel Martzluff (2005), témoigne de l'estime et de l'attachement affectueux que ses confrères, amis et connaissances lui portaient.

Dominique SACCHI 


\section{RÉFÉRENCES}

CAMPMAJO P. 2012 - Ces pierres qui nous parlent. Les gravures rupestres de Cerdagne (Pyrénées Catalanes) des Ibères à l'époque contemporaine, Trabucaire, 642 p., fig.

DESCAMPS C. 2005 - Jean Abélanet, premier professeur de Préhistoire à Perpignan, In : M. Martzluff (dir.), actes du colloque Roches ornées roches dressées. Les hommes et leurs terres en Pyrénées de l'Est, actes du colloque en hommage à Jean Abélanet, université de Perpignan, 2001, A.A.P.O.- Presses universitaires de Perpignan, p. 39-40.

LALLEMAND V. (dir.) 2019 - Catalogue de l'exposition Mémoires de pierres. Les gravures rupestres de Cerdagne et d'ailleurs, Château-Musée de Bélesta, non pag., fig., pl.

MARTZLUFF M. (dir.) 2005 - Roches ornées, roches dressées. Aux sources des arts et des mythes. Les hommes et leurs terres en Pyrénées de l'Est, actes du colloque en hommage à Jean Abélanet, AAPO - Presses universitaires de Perpignan, 574 p., fig.

SACCHI D. 1984 - Catalogue de l'exposition L'art paléolithique de la France méditerranéenne, Musée des BeauxArts, Carcassonne, 52 p., fig.

SACCHI D. 1986 - Le Paléolithique supérieur du Languedoc occidental et du Roussillon, Gallia-Préhistoire, XXle supplément, C.N.R.S. 1986, 284 p., fig., pl. h.-t., tabl., dépl.

SACCHI D. 1991 - Le Solutréen des Pyrénées méditerranéennes françaises et de leurs abords, In : Kozlowski J. K. actes du colloque "Feuilles de pierre. Les industries à pointes foliacées du paléolithique supérieur européen », Cracovie, 1989, ERAUL,

\section{BIBLIOGRAPHIE SÉLECTIVE DE JEAN ABÉLANET *}

\section{Ouvrages}

ABÉLANET J. 1977 - Les gravures rupestres du Roussillon, Thèse $3^{e}$ cycle, Université de Montpellier, vol. 1, Roches à cupules et gravures schématiques d'ambiance dolménique, p. 1-121, pl.; vol. 2, Les gravures schématiques linéaires, p. 122-201, pl.

ABÉLANET J. 1986 - Signes sans paroles : cent siècles d'art rupestre en Europe occidentale, Hachette, 345 p., fig., pl. h.-t.

ABÉLANET J. 1990 - Les roches gravées nord-catalanes, Centre d'Études Préhistoriques Catalanes et Terra Nostra, 212 p., fig., tabl.

ABÉLANET J. 1992 - Autrefois des hommes... Préhistoire du pays catalan, Trabucaire, 206 p., fig., tabl.

ABÉLANET J. 2011 - Itinéraires mégalithiques. Dolmens et rites funéraires en Roussillon et Pyrénées nord-catalanes, Trabucaire, 349 p., fig.

ABÉLANET J. 2017 - L'homme et le cerf, préhistoire d'un mythe, Trabucaire, 170 p., fig.

\section{Livret-guide}

ABÉLANET J. 1982 - Le Musée de Tautavel. Livret-Guide, Conflent éd., 60 p., fig.

\section{Articles, communications, contributions à des ouvrages}

ABÉLANET J. 1951 et 1953 - Ossuaires énéolithiques dans les Corbières Roussillonnaises, Études Roussillonnaises, t. 1-2, p. 128-133, fig. ; t. 3-1, p. 7-14, fig.

ABÉLANET J. 1960 - Ossuaires chalcolithiques des Pyrénées-Orientales, Annales t. 9-3, Travaux de l'Institut d'art préhistorique de Toulouse, p. 5-17, fig.

ABÉLANET J. 1961 - Les gravures schématiques linéaires des Pyrénées-Orientales, Annales, t. 10-3, Travaux de l'Institut d'art préhistorique de Toulouse, p. 5-7.

ABÉLANET J. 1962 - Permanence d'un art schématique dans les Pyrénées-Orientales, Annales, t. 11, Travaux de l'Institut d'art préhistorique de Toulouse, p. 5-17.

ABÉLANET J. 1966a - Les gravures rupestres schématiques des Pyrénées-Orientales, In : Actes $d u$ XVIII Congrès préhistorique de France, Ajaccio, p. 393-398, fig.

ABÉLANET J. 1966b - Les plus vieilles archives des Pyrénées-Orientales : les roches gravées préhistoriques, Bulletin des Archives des Pyrénées-Orientales, n³1, p. 2743, fig.

ABÉLANET J. 1969 - Les couples humains dans l'art schématique des Pyrénées-Orientales, I.PE.K., p. 30-33, fig.

ABÉLANET J. 1970a - Les dolmens du Roussillon, in Actes du Colloque les civilisations néolithiques du Midi de la France - Atacina n5, p. 74-79, 2 fig.

ABÉLANET J. 1970b - Une tombe néolithique : l'Arca de Calahons (Catlla, Pyrénées-Orientales), In : J. Guilaine (dir.), Actes du Colloque les civilisations néolithiques du Midi de la France, Narbonne 1970 - Atacina, n5, p. 54-55, fig. 42, p. 223-237, fig.

ABÉLANET J. 1976a - Essai de datation de l'art schématique linéaire méditerranéen, in Actes du IXe Congrès de I'U.I.S.P.P. Colloque XXVII, Nice 1976, p. 69-73, fig.

ABÉLANET J. 1976b - l'Art schématique linéaire, in H. de Lumley, M.-E. Fonvielle et J. Abélanet (dir.),Vallée des Merveilles, Livret Guide de l'excursion C1, IXe Congrès de I'U.I.S.P.P., Nice, 1976, p. 137-170.

ABÉLANET J. 1976c - Les gravures du Val des Merveilles et le symbolisme du taureau en Méditerranée, In : H. de Lumley, M.-E. Fonvielle et J. Abélanet (dir.),Vallée des Merveilles, Livret- Guide de l'excursion C1, IXe Congrès de I'U.I.S.P.P., Nice, 1976, p. 47-118.

ABÉLANET J. 1976d - Les roches gravées du Capcir et de la Cerdagne (Roussillon), In : Actes du 1er colloque International d'Archéologie de Puigcerda, 1974 - Cypsela $n^{\circ} 1$, p. 79-82, fig.

ABÉLANET J. 1979 - El poblament prehistoric del midgia pirenenc, Annals del Centre de Perpinya de l'Institut d'Estudis Andorans, Université de Perpignan, p. 9-19. 
ABÉLANET J. 1983 - Les temps d'avant l'Histoire, In : Jean Sagnes (dir.), Le pays catalan, t. 1, Société nouvelle d'Éditions régionales et de diffusion, Pau, p. 20-69, fig.

ABÉLANET J. 1984 - Pierres droites, pierres plantées du Roussillon : bornes ou menhirs?, In : Actes du $106^{\circ}$ Congrès national des Sociétés savantes, section archéologie, Perpignan, 1981, p. 21-38, fig.

ABÉLANET J. 1985 - Le premier site d'art rupestre paléolithique à l'air libre : le rocher gravé de Campôme, Conflent, $n^{\circ} 133$, p. 2-7, fig.

ABÉLANET J. 1987a - Dolmens et rites funéraires en Roussillon, Conflent, n¹45, p. 2-25, fig.

ABÉLANET J. 1987b - Le site à gravures rupestres du Valat de la Figuerassa (Commune de Tarerach, PyrénéesOrientales), Bulletin de la Société méridionale de spéléologie et de préhistoire, t. 27, p. 33-67, fig

ABÉLANET J. 1987C - Pierre Ponsich, préhistorien, In : Etudes roussillonnaises offertes à Pierre Ponsich, Le Publicateur, p. 15-16.

ABÉLANET J. 1989 - Aperçus sur l'art rupestre et les figurations religieuses des civilisations néolithiques de l'Europe occidentale, in Actes du Colloque La sculpture rupestre en France de la Préhistoire à nos jours, Brantôme 1988 - Bulletin de la Société historique et archéologique du Périgord, n¹16, p. 115-125, fig.

ABÉLANET J. 1990 - Bilan de dix années de recherches sur les sites paléolithiques de plein air de la vallée de Tautavel-Vingrau, Travaux de Préhistoire catalane, $\mathrm{n}^{\circ} 6$, p. 17-36, fig.

ABÉLANET J. 1993 - Restauration du dolmen dit Balma de $\mathrm{Na}$ Cristiana (l'Albère), Bulletin de l'Association Archéologique des Pyrénées-Oles, nº, p. 75-78, fig.

ABÉLANET J. 1996 - La Fenollèda : els precedents antics : de la Prehistoria a la fi del mon iberic-roman i visigot, El Donasà, la Fenolleda, el Perapertusès, Catalunya Romanica, t. 25, Barcelone, p. 277-283.

ABÉLANET J. 2003a - Le bloc gravé de Railleu, Regard sur 20 ans d'archéologie en Roussillon, Bulletin de l'Association Archéologique des Pyrénées-Oles, n¹8, p. 131132, fig.

ABÉLANET J. 2003b - Les roches à entailles ou pseudopolissoirs des Pyrénées catalanes et leur rapport avec le style rupestre linéaire, In : Juan-Ramon Gonzales i Pérez

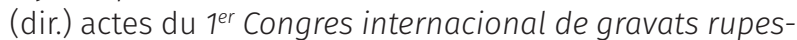
tres i murals, Homenatge a Luis Diez-Coronel, Lleida, 1992, Institut d'Estudis Ilerdencs, p. 595-618, fig.

ABÉLANET J. 2004 - Essai d'interprétation des roches à intailles du Roussillon et des Pyrénées catalanes, Bulletin de l'Association Archéologique des Pyrénées-Oles, n¹9, p. 79-82, fig.

ABÉLANET J. 2005 - Le thème du damier dans l'art rupestre linéaire des Pyrénées catalanes et ses liens avec les cultes anciens, In : M. Martzluff (dir.) Actes du colloque Roches ornées, roches dressées, 2001, Presses Universitaires de Perpignan, p. 207-217, fig.
ABÉLANET J. 2007 - Collaboration à J. Kotarba, G. Castellvi, F. Mazière (dir), Carte archéologique de la Gaule, Les Pyrénées-Orientales (CAG 66), Académie des Inscriptions et Belles-Lettres, 2007, p. 199 - 621.

ABÉLANET J. 2009 - L'art schématique protohistorique catalan : du semi-naturalisme à l'abstraction. In : De Méditerranée et d'ailleurs, Mélanges offerts à Jean Guilaine, Archives d'Ecologie Préhistorique, 2009, p. 11-18, fig.

ABÉLANET J. 2012 - La tuile peinte de Tautavel et la permanence d'un art schématique dans les PyrénéesOrientales, In : M. Martzluff, A. Catafau, M. Galinier (dir.), Tautavel. Des hommes dans leur vallée, Presses Universitaires de Perpignan, p. 545-551, fig.

ABÉLANET J., CHARLES R.-P. 1964 - Un site du Néolithique ancien en Roussillon: La Cova de l'Esperit (habitat et sépultures), Cahiers Ligures de Préhistoire et d'Archéologie, n० 13, p. 177-206, fig.

ABÉLANET J., CHARLES R.-P. 1964b - L'ossuaire des gorges du Verdouble près de Tautavel (Pyrénées-Orientales), Cahiers Ligures de Préhistoire et d'Archéologie, $n^{\circ} 13$, p. 228-236, fig.

ABÉLANET J., CHARLES R.-P., RIGAUD L. 1964 - L'ossuaire chalcolithique de Roquefort-des-Corbières (Aude), Cahiers Ligures de Préhistoire et d'Archéologie, t. 13, p. 237-250, fig.

ABÉLANET J., DESCAMPS C. 1999 - Les premières recherches à la Cauna de l'Arago (Tautavel), Préhistoire ariègeoise, t. 54, p. 5-14.

ABÉLANET J., GUAL R. 1969 - Le dolmen de la Lloseta (ClaraVillerach, Pyrénées-Orientales), Atacina n 4, p. 27-31, fig.

ABÉLANET J., GUILAINE J., VAQUER J., AYMÉ R. 1988 - LeS gravures schématiques de la Cauna de Perillos, PyrénéesOrientales, Bulletino del centro Camune di studi Preistorici, $n^{\circ} 24$, p. 117-121, fig.

ABÉLANET J., MARTZLUFF M. 1989 - Note sur la découverte d'un campement de plein air de l'homme de Tautavel près de la Cauna de l'Arago, Travaux de Préhistoire catalane, $n^{\circ} 6$, p. 37-41, fig.

ABÉLANET J., MARTZLUFF M., BLAIZE Y. 1987 - Le Paléolithique des Pyrénées-Orientales. État de la recherche et nouvelles données, Bulletin de l'Association Archéologique des Pyrénées-Oles, n4, p. 2-23, fig.

CLAUSTRE F., MARTZLUFF M., ABÉLANET J., DONAT r., THEILLOL V. 2000-2001 - La Coma de Janicot (Salses-PyrénéesOrientales), Études Roussillonnaises (nouvelle série), t. 18, p. 63-78, fig.

GUILAINE J., ABÉLANET J. 1964- Esquisse chronologique de l'âge du Bronze dans les Pyrénées-Orientales, Cahiers Ligures de Préhistoire et d'Archéologie, n 13, p. 207-227, fig.

GUILAINE J., ABÉLANET J. 1966 - La céramique poladienne du Roussillon et du bassin de l'Aude dans son contexte méridional, in Actes du IV Symposium de Prehistoria Peninsular, Pampelune, 1965, p. 129-148, fig. 
GUILAINE J., ABÉLANET J., BOUISSET P., CLOTTES J. 1969 Datations radiocarbone du Languedoc occidental et du Roussillon, Atacina n ${ }^{\circ}$, p. 50-61.

GUILAINE J., ABÉLANET J., MARTZLUFF M. 1981 - Prehistoria d'Andorra - Primera campanya d'excavacions (1979) à la Balma de la Margineda, Annals del Centre de Perpinya de l'Institut d'Estudis Andorrans, Université de Perpignan, p. 7-13, fig.

GUILAINE J., VAQUER J., BARRIÉ P., ABÉLANET J. 1974 - Le gisement de la Balma de Montbolo et les recherches de 1969-1970, In : J. Guilaine (dir.) La Balma de Montbolo et le Néolithique de l'Occident Méditerranéen, Institut Pyrénéen d'Études anthropologiques, Toulouse, p. 19-46, 20 fig.

LUMLEY H. (de), COLLINA-GIRARD J., ABÉLANET J., BAZILE F., MEIGNEN L. 1976 - Les premières industries humaines en Languedoc méditerranéen et en Roussillon, In : La Préhistoire française, CNRS édit., t. 2, p. 777-794.

LUMLEY H. (de), FONVIELLE M.-E., ABÉLANET J. 1976 - Les gravures rupestres de l'âge du Bronze dans la région du mont Bego (Tende, Alpes-Maritimes), in La Préhistoire Française, t. 3, CNRS édit., p. 222-236, fig.

MARTZLUFF M., ABÉLANET J. 1987 - La Cova de l'Esperit : bilan des dernières recherches et nouveaux apports sur le Mésolithique et le Néolithique des Pyrénées-Orientales, Études Roussillonnaises offertes à Pierre Ponsich, Le Publicateur, Perpignan, p. 99-113, fig.

MARTZLUFF M., ABÉLANET J. 1989 - Note sur la découverte d'un émoussé de schiste gravé du Paléolithique supérieur dans la couche 3 de la Cova de l'Esperit (Salses, PyrénéesOrientales), Bulletin de la Société Préhistorique française, t. 86-5, p. 143-145, fig.
MARTZLUFF M., ABÉLANET J. 1990 - Le campement magdalénien du Rec del Penjat (Commune de Vingrau, Pyrénées-Orientales), Travaux de Préhistoire catalane, $n^{\circ} 6$, p. 43-61, fig.

MARTZLUFF M., ABÉLANET J. 1995 - La station mésolithique de Lo Garriga à Espira-de-l'Agly (Pyrénées-Orientales), Travaux de Préhistoire catalane, n²8, 1992/1995, p. 13-19, fig.

PARDO J., CURA M., ABÉLANET J. 1975 - Sepulcros megaliticos de la Cerdanya y del Capcir, Corpus de sepulcros megaliticos, Barcelone, 30 p., fig.

SACCHI D., ABÉLANET J. 1980 - Quelques données archéologiques sur le peuplement paléolithique supérieur du Conflent, In : Conflent, Vallespir et montagnes catalanes, Actes du $\mathrm{LI}^{\circ}$ Congrès de la Fédération Historique du Languedoc méditerranéen et du Roussillon (Prades et Villefranche-de-Conflent), 1978, CTHS p. 11-29, fig, tabl.

SACCHI D., ABÉLANET J., VILETTE Ph. 1984 - Cova Bastéra, in L'art des cavernes. Atlas des grottes ornées paléolithiques françaises, Ministère de la Culture, p. 347-349, fig.

SACCHI D, ABÉLANET J., BRULÉ J.-L., MASSIAC Y., RUBIELLA C., VILETTE P. 1988 - Les gravures rupestres de Fornols-Haut, Pyrénées-Orientales, L'Anthropologie, t. 92, nº1, p. 87-100, fig

* Liste dressée à partir de la bibliographie exhaustive établie par Michel Martzluff. 